\title{
Pytch - an environment for bridging block and text programming styles (Work in progress)
}

\author{
Glenn Strong \\ Trinity College Dublin \\ Ireland \\ Glenn.Strong@scss.tcd.ie
}

\author{
Ben North \\ Trinity College Dublin \\ Ireland \\ Ben.North@tcd.ie
}

\begin{abstract}
Block-based programming languages, and Scratch in particular, are widely used to introduce young learners to programming. As these students progress through their education, they want or need to transition to using text-based systems and encounter a number of challenges as they do so. Issues with syntax, text editing, and memorisation are all significant, but the change of programming paradigm is also a challenge. This paper discusses the design and development of a system to help students make the transition to text-based programming environments more easily.

Sprites, animations and sound form the basis of most Scratch programs and these engaging features become unavailable at the same time as students are facing transition difficulties related to text editing and program structure. From programs designed in an actorbased event-driven system with easy concurrency, students have to move to procedural or class-based programs where multimedia features are accessed quite differently and programs are designed around explicit event loops.

In this paper we introduce a new programming system, Pytch, which embodies "Scratch-Oriented programming" in Python. Using a web-based environment that requires no local setup, students can build Python programs using the familiar sprites and concurrent event-driven model learned in Scratch. The system offers the programming model inspired by Scratch through a Python library and a runtime augmented with a form of managed concurrency.

The motivation and related work are discussed, and the system is presented in its current form. The next stage will be to evaluate the effectiveness of the system with users.
\end{abstract}

\section{CCS CONCEPTS}

- Social and professional topics $\rightarrow$ Computing education; • Applied computing $\rightarrow$ Interactive learning environments.

\section{KEYWORDS}

Education, Programming languages, Scratch, Games, Python

\section{ACM Reference Format:}

Glenn Strong and Ben North. 2021. Pytch - an environment for bridging block and text programming styles (Work in progress). In The 16th Workshop in Primary and Secondary Computing Education (WiPSCE '21), October 18-20,

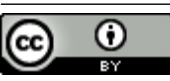

This work is licensed under a Creative Commons Attribution International 4.0 License.

WiPSCE '21, October 18-20, 2021, Virtual Event, Germany

(c) 2021 Copyright held by the owner/author(s).

ACM ISBN 978-1-4503-8571-8/21/10.

https://doi.org/10.1145/3481312.3481318
2021, Virtual Event, Germany. ACM, New York, NY, USA, 4 pages. https: //doi.org/10.1145/3481312.3481318

\section{INTRODUCTION}

Block-based languages are widespread as introductory tools in computer programming [25], both in classrooms and informal environments such as Code.org and CoderDojo. Block-based languages provide excellent support for introductory programming, but after some time students generally find they want, or are required, to move to text-based languages [10]. At this point, generally, a number of issues arise related to this transition [16, 24].

In this paper we describe a new programming environment, "Pytch", designed to support students in making the transition from Scratch to Python. Unlike some other attempts to support students in this activity Pytch does not focus on the question of using a text editor. The novel contribution of Pytch is that it focuses on the question of programming paradigm, allowing students to continue to write the style of program they have become familiar with. The choice of programming languages is driven by popularity; both are very widely used in programming education, and it is common to find Python being used as a first text-based language for users transitioning from Scratch [22].

\section{MOTIVATION}

The issues that arise in transition from Block-based programming to text-based programming have been well documented [16, 19, 23]. When considering the question of how to reduce the challenges faced by users we can loosely think of these difficulties as being in three categories:

- Issues that are inherent in the semantics of the programming language being learned. Such issues include understanding type systems, concepts such as variable references and aliasing, and so on.

- Issues that are part of the language but which a well designed programming environment can ameliorate, such as difficulty mastering language syntax in all its forms (remembering keywords and various syntactic forms, getting the precise placement of punctuation correct, managing code structure and indentation, and so on), memorising the large number of functions and commands available, and so on.

- Issues related to the new programming paradigm. For example, the move from an object-based language such as Scratch to a class-based language like Python. Under this heading we also consider the move from designing Actor model programs of the sort supported by Scratch to designing procedural console-oriented programs as typically introduced with Python. We can think of this category as relating to 
what users are doing with the language, and what affordances it offers.

In general, the very tight coupling between language and environment in systems such as Scratch makes the distinction between language and environment uninteresting, but this is not true for languages like Python. As a result we can consider what sort of programming environment could help support transition to the language.

Our goal with Pytch is to focus on the third category of challenge, by providing an environment that allows users to write Python programs that make use of what they have learned about writing the games and other activities that provide highly motivating activities as found in Scratch.

\subsection{Challenges with changing paradigm}

Users transitioning from block-based environments experience a significant loss of confidence when doing so $[18,19]$. When students are engaged in the process of transitioning to text-based systems, they have not only to overcome the difficulties of programming in text, but they have also to re-think their entire conception of how a program is organised. Instead of working with communicating concurrent objects, their program might, for example, consist of a batch-like sequence of console input/output operations which can reduce motivation and a sense of fun [12].

As another example, we can imagine the typical experience of a student familiar with Scratch attempting to write a game in Python for the first time; they must tackle not only language syntax and editing issues but also learn how to organise sound and video assets in the filesystem, manage source files, and so on. Again, they can no longer work with a set of objects independently processing user events. The program must be organised around an explicit event pump and the programmer must manually dispatch events, and since concurrency is not available the user must take responsibility for ensuring the various parts of the program interleave their behaviour [26, ch.17].

\subsection{Related work}

Many systems have been produced to help users overcome issues in block-text transition. For the sake of brevity here we will focus on programming environments that have a particular relevance to this work. Particularly we omit discussion of more purely pedagogical approaches.

A number of systems address the syntax-related problems of transfer by defining block-editing environments that target high level languages (either of their own design, or established languages such as Python, JavaScript or Java).

The Alice [11] language provides a block-based editor and a 3D "microworld" that allows users to tell stories. Alice is a distinct programming language though it is designed to facilitate a transition into Java programming, and terminology and function names follow the model of the Java APIs rather than the simpler Scratch framework.

Stride [16] (and the associated Greenfoot API [15]) combine block and text based systems with an innovative Frame-based editing system. The system has easy primitives for making games, but the design is again oriented towards transitioning users into Java. In particular Greenfoot offers a familiar programming model with Actor behaviour. The use of Java makes the system more attractive for older users [15].

Robinson's Patch [20] takes a different approach, modifying the Scratch environment to present a more Python-like syntax. The underlying language is still Scratch, and it is proposed as a way to better support the learning of fundamental principles, and to assist in transition to Python.

The Edublocks [5] browser-based environment offers a subset of Python with block-based (and optional text based) editing and a selection of Python libraries. Similarly, MakeCode Python [7] has similar editing capabilities and supports the Static Python (SPY) subset of the language.

A number of solutions implemented as libraries take a similar tack; makeblock [2] has some support for displaying a block-based program as Python, though the generated code uses a number of unusual techniques. Blocklike [4] offers a JavaScript interface with a strong relationship to Scratch, including a concurrent Actor model (although thread yielding must be done manually).

None of these alternatives are strict in following our approach of carrying the Scratch style programming model through to the text-based experience, and thus they all require all the user to "unlearn" [20] how to structure a program.

\section{3 РYТСН}

Our proposed solution is a new environment which brings a familiar Scratch-style programming model to the Python language.

\subsection{Design principles}

We identified a number of key design principles:

DP1 : Support the Scratch concurrent programming model.

DP2 : Support the Scratch "micro-world" of sprites, costumes, sounds, and so on [13].

DP3 : Where consistent with DP1 we want to encourage the use of idiomatic Python code (commonly called "Pythonic" code).

DP4 : Minimise the need for complex set-up and administration.

DP5 : Support user autonomy via integrated learning materials.

Our core goal with Pytch is to allow users to begin learning about Python development without forcing a completely new paradigm for structuring programs, or completely new domains of programs to write. DP1 addresses the issue of paradigm [16], allowing users to make use of what they have already learned about designing and structuring programs in Scratch. DP2 supports creating the engaging content familiar to Scratch programmers [12].

DP3, by contrast, is motivated by the desire to support users in eventually gaining fluency in both reading and writing Python programs. Users who wish to move beyond the Scratch-style programming model will encounter other Python projects, libraries, and sample code and having exposure to a consistent style from the start should help this transition [17]. This principle supports the idea that we are encouraging users to develop an understanding not only of the surface-level syntax of Python but of how best to work in that language to solve problems.

It is normal to distinguish general purpose programming techniques (sometimes called "design patterns") from idiom (language specific techniques). The Python community has an unusually 
strong notion of programming idiom, and the term "Pythonic" is frequently used to describe this widely accepted approach [9].

DP4 relates to simplifying access to the environment. Making it as easy as possible to access the environment and begin coding will reduce frustration for users. In a classroom context teachers who may lack dedicated IT support and a simple way to install environments with complex dependencies will also benefit [22].

DP5 relates to the use of the environment for learning; it is not enough to provide the programming environment, students must also be able to access resources that will encourage and support them in learning how to work with it. Documentation and guided tutorials are needed [14, 22].

\subsection{Implementation strategy}

In so far as possible we implement Pytch in pure Python. However, DP1 and DP2 taken together demand that we introduce one significant semantic change from traditional Python programming, and an accompanying IO system that cannot be implemented in Python alone.

Since we wish to support the Scratch programming style (DP1 \& DP2) we must support the underlying semantics, and while much of this can be implemented in pure Python, some lower-level support is needed. Specifically, to fully support the Scratch paradigm, we need to provide support for the kind of actor-model concurrency that Scratch users have become accustomed to.

To support DP4 we have chosen to implement Pytch as a webbased programming environment. For the Python implementation we use a fork of the Skulpt Python transpiler [8], augmented to support cooperative multithreading. Skulpt provides an almostcomplete implementation of Python 3, with good performance.

To this we have added a runtime environment that supports graphics and sound output, and keyboard and mouse input (implemented in HTML5), and a managed multithreaded environment and event model that closely matches that of Scratch.

In the current implementation the user's programs and assets (sounds and images) are retained in browser-local storage. No user data is transmitted to the Pytch servers. Programs can be exported and imported as zip files for transfer to other computers (for example, for a friend to play or for a teacher to assess).

We have modified the Skulpt compiler phase to recognise when it is compiling a Pytch program and to automatically insert thread yielding statements at strategic points. So far as is practical we follow the model of the Scratch runtime here, yielding thread control once per loop body iteration, and other carefully selected points [1] Users can also annotate functions to indicate that loops should be allowed to run to completion without yielding, though the library has been designed to minimise the need for this.

Following DP5 we provide online help and documentation on the Pytch libraries, and a set of guided step-by-step tutorials built into the system help users become familiar with writing Pytch programs. Users are encouraged to explore the system and make their own games and other programs. The design of the Pytch API and the tutorial content are carefully chosen to support DP3.

The environment is shown in figure 1 . The broad structure of the environment will be instantly familiar to our target audience of experienced Scratch users. The image shows the text of the program

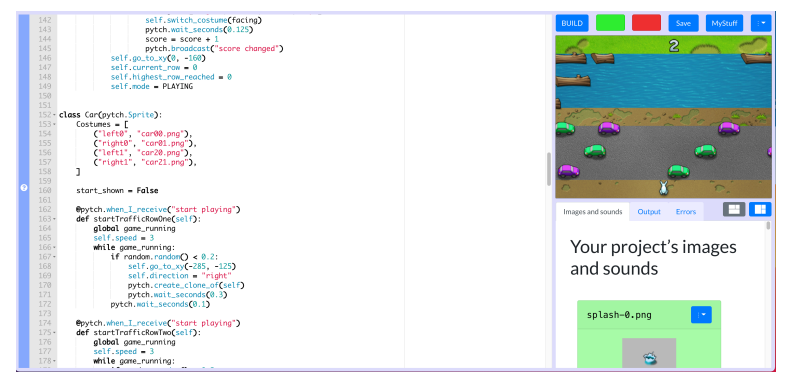

Figure 1: The Pytch IDE with the 'Bunner' tutorial running ${ }^{1}$

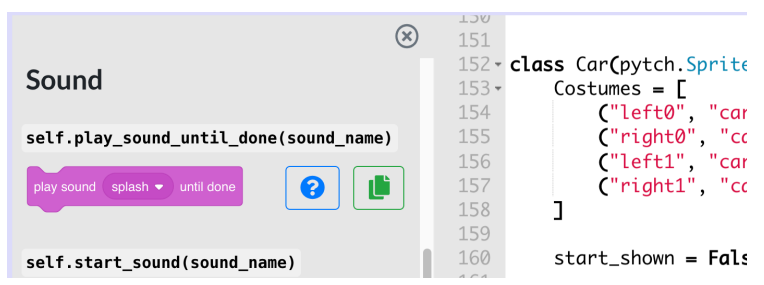

Figure 2: Part of the "method palette" showing correspondence between Pytch methods and Scratch blocks

on the left, the projects graphics and sounds at the bottom right (along with a console and an error reporting tab). At the top-right is the canvas where the stage and sprites are drawn. In the running example each car and log sprite manages its own movement code in a separate (simulated) thread in the Pytch runtime.

Programs are entered using traditional text-editing (we currently use the Ace text editing component [3]), with auto-completion and pop-up function documentation to help users overcome the need to remember what functions are available. Additional help with recall of commands is available via a "method palette" listing Pytch methods and their corresponding Scratch constructs. The palette is displayed using the "help" button to the left of the text editor. Within the palette, buttons can be used to show more detailed help and to copy sample code for pasting to the user's program (figure 2).

\subsection{Language design}

Our desire to support students who are familiar with Scratch leads us to design the Pytch programming interface to closely model the structure of a Scratch program.

Sprites are created by declaring Python classes which inherit from a base pytch. Sprite class; similarly, the stage is declared by a class which inherits pytch. Stage. To match the behaviour of Scratch, the Pytch libraries automatically instantiate objects for all such declared classes. Sprite clones are represented by further instances of the Sprite classes. We collectively refer to sprites and the stage as actors.

Any actor can respond to an event by annotating one or more of its methods; Python decorators are used for this, with decorator names chosen to exploit the assumed familiarity with Scratch "hat

\footnotetext{
${ }^{1}$ Graphics are taken from the Raspberry Pi Foundation book "Code the Classics", used under a Creative Commons Attribution-NonCommercialShareAlike 3.0 Unported (CC BY-NC-SA 3.0) license.
} 


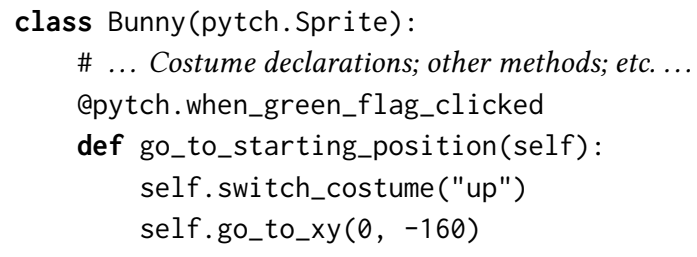

Figure 3: An example of Pytch code

blocks". Figure 3 shows a fragment of Pytch code, with a sprite declaration and a method which will be run in response to an event (in this case, the user clicking the "green flag" element in the Pytch UI to start the program running). Currently supported events include green flag events, key press events, and mouse click events. Events can also be triggered programmatically using the familiar broadcast-and-receive mechanism.

Concurrency in Pytch is accessed via these event responses. A new simulated thread is launched for each method that responds to an event. Threads yield via a library routine, but users should never have to explicitly invoke this; as noted in section 3.2 such calls are automatically inserted at selected points in the user's program.

\subsection{Availability}

Pytch is live at https://pytch.org/, and its code is available under a free-software licence at https://github.com/pytchlang/.

\section{CONCLUSIONS AND FUTURE WORK}

We have developed a system that allows users to begin the process of learning text-based programming with Python by developing interactive programs of the sort that are familiar to Scratch users. The programming environment includes a number of features intended to support users as they begin the process of learning text-based programming.

The design of the Pytch Python API is such that concepts in common Python libraries such as the Flask web framework [6] which makes use of decorators and an event driven paradigm should be familiar, providing a potential pathway for users to continue their progress with Python.

The environment need not rely on pure text entry, and we anticipate experimenting with the program-entry part of the environment and hope to offer some easier-to-use editing tools in the future. Two options actively being investigated are a block-based Python code editor [21] and a frame-based editor in the style of Stride but targeting Python.

We have begun evaluation of Pytch with volunteer users, and early feedback has been positive. The next stage is to conduct full scale trials to evaluate the effectiveness of the environment at supporting users in moving to text-based programming. It is planned to use the environment in short structured courses, evaluating the users' progress with both learning outcomes and confidence with text-based programming. Further development work is also ongoing and we expect to be able to make useful contributions in both formal and informal educational contexts.

\section{REFERENCES}

[1] 2017. Scratch discussion forum. https://scratch.mit.edu/discuss/topic/251360/ ?page $=1$ \#post -2576750

[2] 2020. mBlock. https://mblock.makeblock.com/en-us/

[3] 2021. Ace - The High Performance Code Editor For The Web. https://ace.c9.io/

[4] 2021. BlockLike.js - bridging the gap between block programming and JavaScript. https://www.blocklike.org/

[5] 2021. EduBlocks. https://edublocks.org

[6] 2021. Flask Documentation. https://flask.palletsprojects.com/en/2.0.x/

[7] 2021. Python in MakeCode. https://makecode.com/python

[8] 2021. Skulpt.org. http://www.skulpt.org/

[9] Carol V. Alexandru, José J. Merchante, Sebastiano Panichella, Sebastian Proksch, Harald C. Gall, and Gregorio Robles. 2018. On the usage of pythonic idioms. In Proceedings of the 2018 ACM SIGPLAN International Symposium on New Ideas, New Paradigms, and Reflections on Programming and Software. ACM, Boston MA USA, 1-11. https://doi.org/10.1145/3276954.3276960

[10] Neil C.C. Brown, Jens Mönig, Anthony Bau, and David Weintrop. 2016. Panel: Future Directions of Block-based Programming. In Proceedings of the 47th ACM Technical Symposium on Computing Science Education. ACM, Memphis Tennessee USA, 315-316. https://doi.org/10.1145/2839509.2844661

[11] Stephen Cooper, Wanda Dann, and Randy Pausch. 2000. Alice: A 3-D tool for introductory programming concepts. f. Comput. Sci. Coll. 15, 5 (April 2000), $107-116$.

[12] Mark Guzdial. 2013. Exploring Hypotheses about Media Computation. In Proceedings of the Ninth Annual International ACM Conference on International Computing Education Research. ACM, San Diego San California USA, 19-26. https://doi.org/10.1145/2493394.2493397

[13] Celia Hoyles, Richard Noss, and Ross Adamson. 2002. Rethinking the Microworld Idea. Fournal of Educational Computing Research 27, 1 (July 2002), 29-53. https: //doi.org/10.2190/U6X9-0M6H-MU1Q-V36X

[14] Filiz Kalelioğlu and Yasemin Gülbahar. 2014. The Effects of Teaching Programming via Scratch on Problem Solving Skills: A Discussion from Learners' Perspective. (2014), 19.

[15] Michael Kölling. 2010. The Greenfoot Programming Environment. ACM Transactions on Computing Education 10, 4 (Nov. 2010), 1-21. https://doi.org/10.1145/ 1868358.1868361

[16] Michael Kölling, Neil C. C. Brown, and Amjad Altadmri. 2015. Frame-Based Editing: Easing the Transition from Blocks to Text-Based Programming. ACM Press, 29-38. https://doi.org/10.1145/2818314.2818331

[17] Orni Meerbaum-Salant, Michal Armoni, and Mordechai Ben-Ari. 2011. Habits of programming in scratch. In Proceedings of the 16th annual joint conference on innovation and technology in computer science education (ITiCSE '11). Association for Computing Machinery, New York, NY, USA, 168-172. https://doi.org/10. 1145/1999747.1999796 Number of pages: 5 Place: Darmstadt, Germany.

[18] Luke Moors, Andrew Luxton-Reilly, and Paul Denny. 2018. Transitioning from Block-Based to Text-Based Programming Languages. In 2018 International Conference on Learning and Teaching in Computing and Engineering (LaTICE). IEEE, Auckland, New Zealand, 57-64. https://doi.org/10.1109/LaTICE.2018.000-5

[19] Kris Powers, Stacey Ecott, and Leanne M. Hirshfield. 2007. Through the looking glass: Teaching CS0 with alice. In Proceedings of the 38th SIGCSE technical symposium on computer science education (SIGCSE '07). Association for Computing Machinery, New York, NY, USA, 213-217. https://doi.org/10.1145/1227310.1227386

[20] William Robinson. 2016. From Scratch to Patch: Easing the Blocks-Text Transition. In Proceedings of the 11th Workshop in Primary and Secondary Computing Education. ACM, Münster Germany, 96-99. https://doi.org/10.1145/2978249.2978265

[21] Glenn Strong, Sean O'Carroll, and Nina Bresnihan. 2018. A Block Based Editor for Python. In Proceedings of the 13th Workshop in Primary and Secondary Computing Education. ACM, Potsdam Germany, 1-2. https://doi.org/10.1145/ 3265757.3265788

[22] Claudia Szabo, Judy Sheard, Andrew Luxton-Reilly, Simon, Brett A. Becker, and Linda Ott. 2019. Fifteen Years of Introductory Programming in Schools: A Global Overview of K-12 Initiatives. In Proceedings of the 19th Koli Calling International Conference on Computing Education Research. ACM, Koli Finland, 1-9. https: //doi.org/10.1145/3364510.3364513

[23] David Weintrop, Connor Bain, and Uri Wilensky. 2017. Blocking Progress? Transitioning from Block-based to Text-based Programming.

[24] David Weintrop and Uri Wilensky. 2015. To block or not to block, that is the question: students' perceptions of blocks-based programming. In Proceedings of the 14th International Conference on Interaction Design and Children. ACM, Boston Massachusetts, 199-208. https://doi.org/10.1145/2771839.2771860

[25] David Weintrop and Uri Wilensky. 2019. Transitioning from introductory blockbased and text-based environments to professional programming languages in high school computer science classrooms. Computers \& Education 142 (Dec. 2019), 103646. https://doi.org/10.1016/j.compedu.2019.103646

[26] Peter Wentworth, Jeffrey Elkner, Allen B Downey, and Chris Meyer. 2015. How to think like a computer scientist: Learning with Python 3. (2015). 